\title{
Efeitos imediatos da terapia auricular em indivíduos hipertensos de uma unidade de pronto atendimento
}

\section{The immediate effects of auricular therapy in hypertensive individuals at an emergency medical unit}

\author{
Ana Paula Marca ${ }^{1}$, Bianca Aparecida Caldas Chiapetti², Karen Andrea Comparin ${ }^{3}$, \\ Fernando Amancio Aragão ${ }^{4}$
}

\section{Resumo}

\begin{abstract}
A hipertensão arterial sistêmica (HAS) se caracteriza por níveis elevados de pressão arterial (PA) de maneira contínua. É uma doença de causas multifatoriais, difícil diagnóstico e com altas taxas de mortalidade. Um método com efeito hipotensor rápido é a terapia auricular por sangria, proveniente da Medicina Tradicional Chinesa (MTC). O objetivo deste estudo foi analisar os efeitos imediatos da terapia auricular em indivíduos hipertensos de uma unidade de pronto atendimento da cidade de Cascavel-PR. Para tanto, foi verificada a PA antes do procedimento (PA inicial), e se estivesse $\geq 140 / 90 \mathrm{mmHg}$, era realizada a técnica de sangria no ponto Erjian auricular. Dez minutos após, aferia-se novamente a PA (PA final). Os resultados demonstraram redução significativa tanto na PA sistólica (PAS) inicial (157,27 $\pm 14,49)$ quando comparada à PAS final $(142,97 \pm 14,01)$, quanto na PA diastólica (PAD) inicial $(99,83$ $\pm 11,26)$ em comparação com a PAD final $(90,66 \pm 8,82)$. Conclui-se que a sangria no ápice da orelha foi capaz de reduzir significativamente a PA nos indivíduos investigados. Entretanto, tornam-se necessários novos estudos neste sentido, a fim de comprovar os reais benefícios da acupuntura sobre a HAS.
\end{abstract}

Palavras chave: Acupuntura. Hipertensão. Medicina Tradicional Chinesa. Sangria.

\begin{abstract}
Systemic arterial hypertension (SAH) is characterized by continuously elevated blood pressure (BP) levels. It is a multifactorial disease, difficult to be diagnosed and presents high mortality rates. One method with a rapid hypotensive effect is an auricular bloodletting therapy from Traditional Chinese Medicine. The aim of this study was to analyze the immediate effects of auricular therapy in hypertensive individuals at an Emergency Medical Unit in the city of Cascavel-PR. To do so, the BP was verified before the procedure (initial BP), and if it was within the required criteria, the bloodletting technique was performed at the Erjian auricular point. Ten minutes later, BP was measured (end BP). A significant decrease in the results for initial systolic BP (SBP) $(157,27 \pm 14,49)$ compared to end SBP $(142,97$ $\pm 14,01)$, as well as for initial diastolic BP (DBP) $(99,83 \pm \mathrm{BP} 11,26)$ compared to end DBP $(90,66 \pm$ 8,82 ) has been shown. Therefore, it is concluded that the bleeding of ear apex has been able to cut down significantly the BP of the examined individuals. Nevertheless, in order to prove the real benefits of acupuncture on SAH further studies in this respect are necessary.
\end{abstract}

Keyword: Acupuncture. Hypertension. Medicine Chinese Traditional. Bloodletting.

1 Fisioterapeuta pela Universidade Estadual do Oeste do Paraná, Cascavel, Paraná, Brasil. E-mail: anapmarca@gmail.com

2 Fisioterapeuta pela Universidade Estadual do Oeste do Paraná, Cascavel, Paraná, Brasil.

3 Mestrado em Letras, Linguagem e Sociedade pela Universidade Estadual do Oeste do Paraná, Cascavel, Paraná, Brasil. Professor do curso de Fisioterapia da Universidade Estadual do Oeste do Paraná, Cascavel, Paraná, Brasil.

4 Doutorado em Ciências do Movimento Humano pela Universidade Federal do Rio Grande do Sul, Porto Alegre, Rio Grande do Sul, Brasil. Professor Associado do Curso de Fisioterapia da Universidade Estadual do Oeste do Paraná, Cascavel, Paraná, Brasil. 


\section{Introdução}

A hipertensão arterial sistêmica (HAS) é uma doença de causas multifatoriais, caracterizada por níveis elevados de maneira contínua da pressão arterial (PA). ${ }^{(1)}$ É considerado hipertenso o indivíduo que apresenta uma PA sistólica (PAS) $\geq 140 \mathrm{mmHg}$ e uma PA diastólica (PAD) $\geq 90 \mathrm{mmHg}$, entretanto, para firmar seu diagnóstico, deve-se constatar medidas repetidas desta PA elevada, em pelo menos três ocasiões. ${ }^{(2)}$

Por se tratar de uma doença muitas vezes assintomática, há uma dificuldade por parte dos indivíduos, na procura pelos serviços de saúde, a fim de obter-se um diagnóstico e aderir ao tratamento. Entretanto, é um importante problema de saúde pública, tendo em vista as altas taxas de morbimortalidade e os elevados custos com o tratamento desta patologia. ${ }^{(3)}$

Estima-se que 7,1 milhões de pessoas no mundo vão a óbito anualmente por causa da pressão sanguínea elevada e que $4,5 \%$ das doenças mundialmente conhecidas sejam causadas pela HAS. A prevalência obtida por análises populacionais que possam afirmar a situação real da doença no Brasil como um todo é ainda desconhecida. Inquéritos populacionais em cidades brasileiras nos últimos vinte anos verificaram uma prevalência de HAS acima de 30\%.(4,5) Estima-se que, por volta de $2025,1,5$ bilhões de pessoas no mundo serão hipertensas. ${ }^{(6)}$

O controle da HAS depende de abordagens não farmacológicas e farmacológicas. As medidas não farmacológicas incluem mudança de estilo de vida, que podem reduzir significativamente os níveis de PA como: controle do peso, dieta equilibrada e com pouca ingestão de sódio, prática de exercício físico, cessação do tabagismo e consumo moderado de álcool. ${ }^{(7)}$ Quanto ao tratamento farmacológico, a decisão terapêutica deve basear-se nos valores da $\mathrm{PA}$, na presença ou não de lesão em órgãos-alvo e de fatores de risco associados. ${ }^{(6)}$
A medicação anti-hipertensiva é uma terapia importante para o tratamento da HAS. Contudo, levando em consideraçao os efeitos colaterais e o receio da eficácia terapêutica, esta terapia está longe de ser satisfatória. Assim, torna-se interessante buscar um tratamento alternativo seguro e menos prejudicial para o controle da HAS. ${ }^{\left({ }^{8}\right.}$

A Política Nacional de Práticas Integrativas e Complementares (PNPIC), publicada na Portaria Ministerial $\mathrm{n}^{\circ}$ 971, de maio de 2006, insere a Medicina Tradicional Chinesa/Acupuntura, Medicina Homeopática, Fitoterapia e Termalismo no Sistema Único de Saúde (SUS). Além disso, estimula estados e municípios a formularem suas políticas de práticas integrativas e complementares. Essas práticas promoveram aos usuários do SUS uma nova alternativa para promoção, manutenção e recuperação da saúde, ${ }^{(9)}$ com menos efeitos colaterais quando comparadas a medicina convencional, ${ }^{(10)}$ além de proporcionar um cuidado contínuo, humanizado e integral à saúde. ${ }^{(9)}$

A acupuntura faz parte dos recursos terapêuticos utilizados pela medicina tradicional chinesa (MTC), sendo um dos métodos mais antigos de tratamento e prevenção de diversas patologias. ${ }^{(11)}$ A Organização Mundial da Saúde (OMS) recomenda a acupuntura, tendo produzido publicações sobre sua eficácia e segurança, capacitação de profissionais, bem como métodos de pesquisa e avaliação dos resultados terapêuticos das medicinas complementares e tradicionais. ${ }^{(12)}$ Mesmo com a aplicabilidade segura e eficaz da técnica, ainda é precária a prática do serviço no SUS, visto que não corresponde a real necessidade da população, sendo pouco divulgada e disponibilizada. ${ }^{(13)}$

Segundo a MTC, a HAS é causada por um desequilíbrio na energia dos meridianos. Não existe tal doença na MTC, mas sim, sintomas semelhantes aos encontrados na HAS. A doença seria resultado da interação entre o agente causador (como fatores climáticos, alimentação incorreta, 
emoções/sentimentos e cansaço físico ou mental) e o indivíduo, resultando em alterações funcionais e orgânicas. Com base nestes princípios, a MTC tem como objetivo restabelecer o equilíbrio das energias no organismo. Tanto no tratamento sistêmico, auricular e/ou emergencial, a acupuntura estabiliza esses sintomas e reduz os fatores de risco para o desenvolvimento de doenças cardiovasculares e suas complicações. ${ }^{(14)}$

A sangria auricular é uma técnica da acupuntura que consiste em um pequeno corte na pele com uma lanceta ou agulha facetada ou até mesmo agulha hipodérmica, que faz perfurações em zonas/capilares do pavilhão auricular, resultando na retirada de uma pequena quantidade de sangue. Este método ajuda na comunicação e drenagem dos canais energéticos, eliminando estagnações e facilitando a circulação do sangue. ${ }^{(15)} \mathrm{O}$ estímulo de pontos hipotensores específicos na aurícula, pode aumentar a ativação do nervo vago com consequente redução da pressão sanguínea e frequência cardíaca, acelerando o fluxo sanguíneo. ${ }^{(16)}$

A HAS é um fator de risco para diversas complicações, sendo o SUS responsável por $45,9 \%$ dos atendimentos de pacientes hipertensos. ${ }^{(17)}$

No município de Cascavel-PR, as equipes das Unidades de Saúde não possuem nutricionistas, psicólogos, fisioterapeutas, acupunturistas, assim como outros profissionais da saúde, que poderiam através da alimentação, terapia psicológica e exercícios terapêuticos, tratar esta doença sem a intervenção medicamentosa ou então, combinando as terapêuticas, auxiliando na melhora mais efetiva do grande número de acometidos. A acupuntura também poderia entrar como um grande aliado no tratamento da HAS, seja emergencialmente e/ou no reequilíbrio físico, emocional e energético que ela pode proporcionar. Sendo assim, este estudo teve por objetivo, analisar os efeitos imediatos da terapia auricular em indivíduos hipertensos de uma Unidade de Pronto Atendimento da cidade de Cascavel-PR.

\section{Materiais e Métodos}

Esta pesquisa caracteriza-se como quantitativa, do tipo quase-experimental. A técnica de tratamento utilizada foi a terapia auricular emergencial, por meio da sangria no ápice da orelha de pacientes hipertensos. Foi desenvolvida na cidade de Cascavel-PR, na unidade de pronto atendimento (UPA) Veneza, que atualmente oferece atendimento adulto e pediátrico. As UPAs fazem parte da Política Nacional de Urgência e Emergência, criada pelo Ministério da Saúde em 2002. Funcionam 24 horas por dia, realizando triagem classificatória de risco, prestando atendimento resolutivo aos pacientes acometidos por quadros agudos ou crônicos agudizados e casos de baixa complexidade. ${ }^{(18)}$

Para a classificação de risco dos pacientes atendidos nas UPAs do município de Cascavel-PR, é utilizado o sistema Manchester durante a triagem inicial, que por meio de critérios clínicos, determina a prioridade de atendimento aos pacientes.

A classificação do sistema organiza-se em: emergente, muito urgente, urgente, pouco urgente e não urgente. ${ }^{(19)}$

Os critérios de inclusão para a presente pesquisa foram indivíduos de ambos os sexos, com idade superior a 18 anos, que estavam aguardando atendimento na UPA Veneza, cuja classificação de risco foi determinada pouco urgente ou não urgente e os níveis da pressão arterial sistêmica apresentavam-se maior ou igual a 140/90 $\mathrm{mmHg}$ e menor que 190/120 mmHg, sem outros sintomas associados na triagem inicial.

Conforme o Sistema Manchester, o paciente pouco urgente é aquele que pode aguardar até duas horas e o não urgente até quatro horas para ser atendido. ${ }^{(19)}$

Foram excluídos indivíduos classificados pelo sistema Manchester como emergente, muito urgente e urgente; apresentando a pressão arterial sistêmica menor que 140/90 $\mathrm{mmHg}$ e maior ou igual a 
190/120 mmHg ou então, maior ou igual a 140/90 mmHg e menor que 190/120 mmHg associada a outros sintomas; idade inferior a 18 anos; presença de traumas físicos; grávidas em qualquer período gestacional; usuários de marcapasso; pacientes hemofílicos e com problemas de coagulação.

A amostra constituiu-se de 36 indivíduos de ambos os sexos, identificados nos critérios propostos que consentiram participar da pesquisa, assinando o termo de consentimento livre e esclarecido (TCLE). A pesquisa foi aprovada pelo Comitê de Ética em Pesquisa com Seres Humanos da UNIOESTE, sob o Parecer $N^{\circ}$ 1.545.275.

A coleta ocorreu entre os meses de junho e setembro de 2016, por meio da aplicação de um questionário elaborado especificamente para esta pesquisa composto de questões abertas e fechadas que tinha o objetivo de obter dados de identificação, tais como, idade, sexo, escolaridade, uso de medicação anti-hipertensiva, uso correto da medicação e prática regular de exercício físico.

Após obter os dados de identificação, a PA foi aferida novamente, desta vez pelo experimentador, sendo este valor considerado como PA inicial (anterior à puntura). Se os níveis ainda se mantivessem dentro dos critérios propostos, o paciente passava pelo procedimento da sangria auricular.

Os seguintes materiais foram utilizados na coleta: esfigmomanômetro (marca P.A. Med com braçadeira em nylon e fecho de metal, calibrado), estetoscópio (marca Premium), algodão, álcool $70 \%$ líquido, luvas de procedimento e lancetas descartáveis para sangria (marca G-Tech).

Para aplicação da técnica foi realizada assepsia comálcool a 70\% em todo o ápice da orelha. A técnica de acupuntura utilizou uma lanceta cuja profundidade alcançada é de cerca de dois milímetros. Desta forma, o ponto de acupuntura auricular chamado Erjian, localizado na extremidade da borda superior da hélice auricular, foi punturado. O procedimento foi unilateral sem distinção de hemicorpo. Após a perfuração do ponto foi provocado um pequeno sangramento e este estancado com algodão seco. A lanceta foi descartada em seguida.

O ponto escolhido segundo a MTC possui efeito hipotensor imediato, sendo indicado em casos emergenciais. Após 10 minutos do final do procedimento, com o indivíduo sentado em repouso, verificou-se novamente a PA, sendo este valor considerado como PA final. Até este momento, não houve administração de fármacos, sendo que, após a experimentação o paciente retornava à sala de espera da UPA para aguardar o atendimento médico.

Considerou-se como variáveis de caracterização da amostra: idade, sexo, escolaridade, uso de medicação anti-hipertensiva, uso correto da medicação e prática regular de exercício físico, e para tais variáveis aplicou-se estatística descritiva. As comparações entre as médias dos níveis de PAS e PAD iniciais e finais foram, inicialmente, operacionalizadas através de um teste de normalidade de Shapiro-Wilk. Após identificar a normalidade dos dados, o teste $\mathrm{t}$ pareado foi adotado para identificar as diferenças, para cada variável entre os momentos de coleta iniciais e finais. Todos os testes foram realizados com auxílio do pacote estatístico Statiscal Package Social Sciences (SPSS)_(version 18.0, SPSS Inc., Chicago, IL) considerando-se nível de significância de $\alpha<0,05$.

\section{Resultados}

De acordo com os critérios propostos, os resultados foram obtidos com 36 indivíduos, sendo $21(58,33 \%)$ do sexo feminino e $15(41,66 \%)$ do sexo masculino. A média de idade foi de $52,69 \pm 15$ anos. Quanto à formação, a amostra foi composta por dois (5\%) indivíduos sem alfabetização, 18 $(50 \%)$ com ensino fundamental incompleto, 10 (28\%) com ensino fundamental completo, três $(8 \%)$ com ensino médio incompleto, um (3\%) com ensino médio completo, um $(3 \%)$ com ensino superior completo, e um (3\%) pós-graduado. 
Do total de participantes, $20(55,55 \%)$ relataram a ingestão medicamentosa anti-hipertensiva e desses, 18 (90\%) afirmaram a correta utilização da terapêutica farmacológica. Sete $(19,44 \%)$ relataram praticar atividade física regularmente, enquanto 29 (80,55\%) afirmaram ser sedentários.

Para demonstrar os resultados da sangria sobre a PA foram elaboradas as figuras 1 e 2 que representam o valor da PAS inicial e final e PAD inicial e final respectivamente.
Nota-se que na figura 1, a PAS inicial $(157,27$ $\pm 14,49)$ reduziu quando comparada à PAS final $(142,97 \pm 14,01)$. Assim como na figura 2, visualizase a redução da PAD inicial $(99,83 \pm 11,26) \mathrm{em}$ comparação com a PAD final $(90,66 \pm 8,82)$.

Em ambas as figuras são constatadas uma diminuição significativa $(\mathrm{p}<0,05)$ da PAS e PAD dez minutos após a técnica da sangria.

Figura 1 - Pressão Arterial Sistólica (PAS) anterior à puntura (inicial) e 10 minutos após (final) dos indivíduos que aguardavam atendimento em uma unidade de pronto atendimento de Cascavel (PR), entre junho e setembro de 2016. $\mathrm{O}$ asterisco representa a significância estatística encontrada entre as médias inicial e final ( $<<0,05$, teste $t$ de Student).

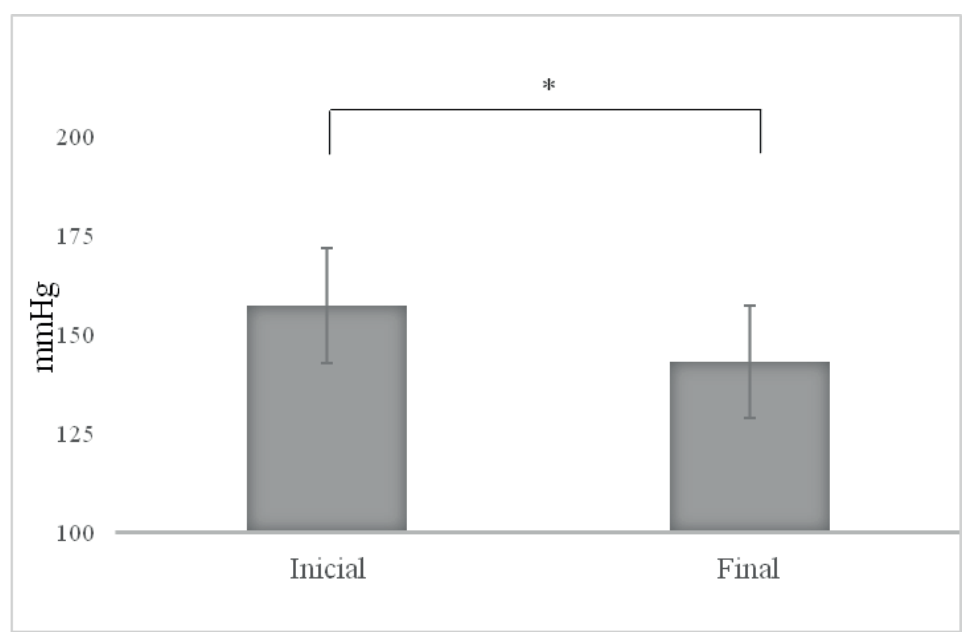

Figura 2 - Pressão Arterial Diastólica (PAD) anterior à puntura (inicial) e 10 minutos após (final) dos indivíduos que aguardavam atendimento em uma unidade de pronto atendimento de Cascavel (PR), entre junho e setembro de 2016. $\mathrm{O}$ asterisco representa a significância estatística encontrada entre as médias inicial e final ( $\mathrm{p}<0,05$, teste $t$ de Student).

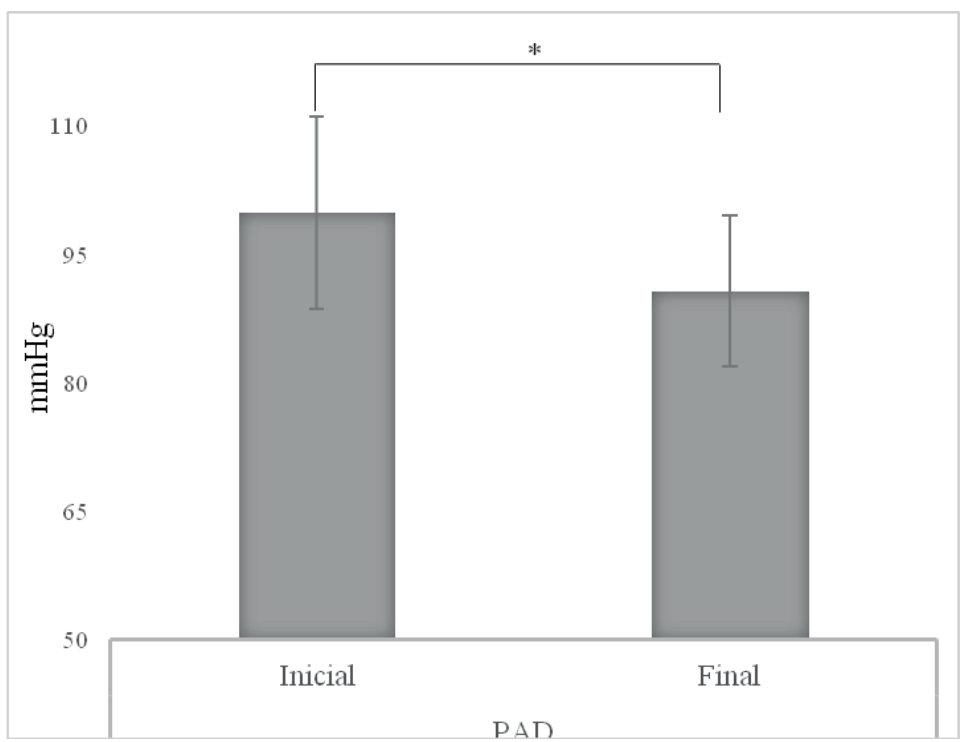




\section{Discussão}

A HAS é considerada fator de risco para o desenvolvimento de doenças cardiovasculares, podendo provocar complicações graves. Por este motivo é reconhecida como problema de saúde pública mundial, acarretando em sobrecarga aos sistemas de saúde pública, devido a elevada taxa de morbimortalidade. ${ }^{(20)}$

O sexo não constitui fator de risco para a HAS. ${ }^{(1)}$ Entretanto ao serem analisados os dados do presente estudo, evidenciou-se uma maior prevalência de mulheres hipertensas $(16,67 \%)$ em relação aos homens.

Quanto a variável idade, sabe-se ser um fator de risco, já que a PA aumenta linearmente com o passar dos anos. ${ }^{(1)}$ Em relação à média da idade $(52,69 \pm$ 15 anos) este estudo diverge de outros autores que identificaram a HAS na população brasileira com mais de 50\% dos casos em indivíduos entre 60 e 69 anos de idade. ${ }^{(21)}$

Metade da amostra do presente estudo não possuía ensino fundamental completo. Este fato pode ser explicado pela desigualdade na saúde, pois, quanto menor o grau de instrução, maior a incidência de complicações da doença devido ao inferior nível de conhecimento e cuidados com a saúde. ${ }^{(22)}$

A prática de exercício físico não é uma realidade vivenciada por grande parte de hipertensos. ${ }^{(7)} \mathrm{A}$ insuficiência da atividade física está intimamente ligada à HAS, tendo como base o resultado de um estudo transversal realizado com 310 hipertensos em um centro de atendimento ambulatorial, em Fortaleza-CE, cuja insuficiência ou ausência de exercício físico ocorreu em $60 \%$ da amostra. ${ }^{(2)}$ Os achados deste trabalho corroboram com os estudos supracitados, onde $80,55 \%$ da amostra informou não praticar exercício físico regularmente.

A prática de exercícios físicos é considerada parte fundamental do tratamento para HAS, visto que a terapêutica anti-hipertensiva por meio do exercício físico, possibilita o controle da PA e influencia positivamente a qualidade de vida dos hipertensos. $^{(23)}$

Neste estudo 55,55\% dos participantes relataram ingestão medicamentosa anti-hipertensiva, e a maioria destes $(90 \%)$ a usavam corretamente. Traçando a mesma linha, um estudo analítico, descritivo e transversal na cidade de Cajazeiras$\mathrm{PB}$, observou que 72,98\% dos 656 hipertensos avaliados eram previamente diagnosticados com HAS e ingeriam medicação para o controle dos níveis pressóricos, desses, apenas 46,91\% tinham a PA controlada. ${ }^{(5)}$

A porcentagem de brasileiros que apresentam HAS controlada, ou seja, que mantêm níveis inferiores a 140/90 mmHg é pequena. Uma possível explicação é que o controle da HAS é advindo de um complexo sistema que inclui aspectos socioeconômicos, culturais, biológicos e de saneamento básico.(24) Outro fator a ser ressaltado, é a inércia terapêutica, explicada como a imprecisão dos profissionais em começar ou ajustar o tratamento medicamentoso quando indicado. ${ }^{(25)}$

A acupuntura pode ser utilizada tanto preventivamente como de modo coadjuvante no tratamento de uma infinidade de patologias, minimizando o sofrimento dos pacientes, atuando no controle das doenças, reduzindo os custos e os efeitos colaterais dos tratamentos convencionais. ${ }^{(26)}$

Entretanto, existem poucos estudos científicos relacionados a acupuntura e/ou sangria sobre a HAS.

Em um ensaio controlado e randomizado realizado em Portugal, com o objetivo de avaliar o efeito da acupuntura no tratamento da HAS, participaram 12 pacientes, seis no grupo experimental e seis no placebo. Em ambos os grupos foi realizado o tratamento em 13 pontos sistêmicos. No grupo experimental foi realizado acupuntura em pontos específicos para o tratamento da HAS, enquanto no placebo, a técnica foi utilizada em pontos que não estão localizados nos meridianos e 
não possuem indicações para a HAS. Verificou-se então, uma redução significativa na PAS no grupo experimental quando comparado ao placebo após a intervenção. $^{(27)}$

Autores sugerem que a acupuntura melhora a hipertensão arterial através de um mecanismo envolvendo alteração cerebral em ratos espontaneamente hipertensos. Este resultado foi obtido através de uma pesquisa experimental na China, envolvendo ratos normotensos e ratos espontaneamente hipertensos. A acupuntura não só diminuiu a PA, como também alterou metabolismo cerebral da glicose no hipotálamo, tálamo, medula oblonga e cerebelo. ${ }^{(28)}$

Uma revisão de literatura concluiu que a acupuntura pode influenciar principalmente em fatores relacionados ao sistema nervoso, estresse oxidativo, sistema endócrino, função cardiovascular e fluxo sanguíneo em ratos hipertensos. Esses resultados podem indicar que a acupuntura é capaz de prevenir complicações por essas vias quando usada no tratamento da HAS. ${ }^{(29)}$

Os achados supracitados indicam possíveis mecanismos envolvidos na redução da PA com a acupuntura. Contudo, esses mecanismos ainda necessitam de apoio científico, vista a necessidade de conhecimento das respostas cardiovasculares e de sua efetividade em pessoas hipertensas.

A auriculoterapia é uma técnica segura e eficaz, de rápida aplicação. ${ }^{(30)} \mathrm{A}$ estimulação da aurícula através da sangria no ápice da orelha é indicada e utilizada nos casos de HAS há mais de 2.500 anos na China. ${ }^{(31)}$

O tratamento com a sangria no ápice da orelha foi eficaz na redução imediata da PA nos indivíduos hipertensos avaliados. Essa efetividade foi visualizada tanto na redução da PAS, quanto na PAD dos participantes.

Um estudo quase-experimental, avaliou as respostas cardiovasculares antes e imediatamente após uma sessão de auriculoterapia (sangria no ápice da orelha associada a estimulação com sementes de mostarda em cinco pontos auriculares), em 10 adultos jovens normotensos do sexo masculino. Como resultado, houve a redução significativa da PAS após aplicação da técnica. ${ }^{(16)}$

Também utilizando a técnica sangria no acuponto Erjian, uma pesquisa quase-experimental realizada em Braço do Norte - SC, contendo 20 voluntários hipertensos e em uso de medicação antihipertensiva, com a PA maior que 120/80 mmHg, obteve a redução da PAS em $80 \%$ dos voluntários. Quanto à $\mathrm{PAD}$, na maioria dos voluntários não se alterou, e em $25 \%$ diminuiu. ${ }^{(15)}$

Desta forma, a técnica da sangria pode ser atribuída ao efeito hipotensor encontrado neste trabalho e nos estudos supraditos. Entretanto, destaca-se a importância e a necessidade da realização de novos ensaios clínicos, que busquem comprovar os mecanismos e efeitos terapêuticos da sangria no ápice da orelha sobre a HAS, podendo assim, garantir à população a possibilidade de uma intervenção segura e eficaz com as terapias complementares no sistema público de saúde, inclusive nas situações de urgência e emergência.

A pesquisa apresenta algumas limitações tais como: a ausência de um grupo controle, que serviria como referência-padrão às variáveis do estudo, o pequeno número da amostra, e a inexistência de avaliadores cegos.

\section{Conclusão}

Aterapia auricular através da sangriano ápice da orelha foi capaz de reduzir significativamente tanto a pressão arterial sistólica, quanto a diastólica nos indivíduos investigados.

Levando em consideração ser uma técnica segura, rápida e de baixo custo, sugere-se novos estudos neste sentido, a fim de avaliar os seus benefícios e desta forma, ampliar a sua oferta no SUS. 


\section{Agradecimentos}

Os autores agradecem à Secretaria Municipal de Saúde de Cascavel (PR) e aos profissionais da Unidade de Pronto Atendimento (UPA) Veneza pela colaboração neste projeto.

\section{Referências bibliográficas}

1 Malachias M, Souza W, Plavnik F, Rodrigues C, Brandão A, Neves M, et al. $7^{\text {a }}$ Diretriz Brasileira de Hipertensão Arterial. Arq Bras Cardiol. 2016;107(3):183.

2 Pereira IMO. Proposta de intervenção interdisciplinar para a adesão dos pacientes ao tratamento da hipertensão arterial sistêmica. Liph Sci. 2015;2(2):21-40.

3 Vieira CPB, Nascimento JJ, Barros SS, Luz MHBA, Valle ARMC. Prevalência referida, fatores de risco e controle da hipertensão arterial em idosos. Ciência, Cuid e Saúde. 2016;15(3):413-20.

4 Nobre F, Coelho EB, Lopes PC, Geleilete TJM. Hipertensão arterial sistêmica primária. Med (Ribeirao Preto Online). 2013;46(3):256-72.

5 Santos A, Barreto V, Oliveira S, Gomes C, Viana K, Brasil B, et al. Perfil Epidemiológico da Hipertensão Arterial Sistêmica na população de Cajazeiras, Paraíba. Rev Bras Ciências da Saúde. 2013;17(3):253-62.

6 Neto VLS, Sucupira PA, Virgolino FSS, Mendonça AEO, Filho JJ. O perfil sócio-epidemiológico dos hipertensos das Unidades básicas de Saúde. Rev da Univ Val do Rio Verde. 2013;10(1):445-56.

7 Oliveira TL, Miranda LP, Fernandes PS, Caldeira AP. Eficácia da educação em saúde no tratamento não medicamentoso da hipertensão arterial. Acta Paul Enferm. 2013;26(2):179-84.

8 Zhao XF, Hu HT, Li JS, Shang HC, Zheng HZ, Niu JF, et al. Is Acupuncture Effective for Hypertension? A Systematic Review and Meta-Analysis. PLoS One. 2015;10(7):1-21.

9 Pereira CF, Villela WV. Organização e funcionamento do serviço de acupuntura na rede pública de saúde. Investigação. 2015;14(1):150-8.

10 Schveitzer MC, Zoboli ELCP. Role of complementary therapies in the understanding of primary healthcare professionals: a systematic review. Rev da Esc Enferm da USP. 2014;48(Esp):184-91.
11 Serpa L, Watanabe L, Carvalho A. Utilização da Acupuntura em Órgãos e Vísceras, Através dos Pontos Bei Shu: Revisão Bibliográfica. Amaz Sci Heal. 2016;4(1):35-40.

12 Ministério da Saúde (BR), Secretária de Atenção à Saúde. Política nacional de práticas integrativas e complementares no SUS: atitude de ampliação de acesso. Brasília: Ministério da Saúde; 2015.

13 Moyses FC. Evolução do serviço de acupuntura no sus: desafios e atualidades [dissertação]. São Paulo (SP): Pontifícia Universidade Católica de São Paulo; 2016.

14 Júnior JCN. Hipertensão arterial sistêmica: um estudo sobre a ótica ocidental e oriental e o uso de fitoterapia e ervas medicinais no Brasil [dissertação]. São José dos Campos (SP): Faculdade de Educação, Ciência e Tecnologia - Unisaúde/CEFIRVAL; 2014.

15 Oliveira RR. Hipertensão Arterial Sistêmica: Efeito Hipotensor da Sangria no Ponto Reflexo Cerebral da Auriculoterapia. Rev Bras Ter e Saúde. 2013;4(1):1-5.

16 Ferreira AP, Rocha TC, Neto AFE, Rodrigues KLS, Aleixo LB, Ramos PS. Respostas cardiovasculares agudas à uma sessão de auriculoterapia em indivíduos normotensos. Rev Bras Ciências Médicas e da Saúde. 2016;4(4):1-7.

17 Chaves DFL, Jesus JH, Geron VLMG, Nunes JS, Lima RRO. A substituição do captopril pelo enalapril no tratamento de hipertensão arterial no âmbito do sistema único de saúde. Saber Científico. 2017;6(2):111-24.

18 Oliveira SN, Ramos BJ, Piazza M, Prado ML, Reibnitz KS, Souza AC. Unidade De Pronto Atendimento Upa 24h: Percepção Da Enfermagem. Texto Context - Enferm. 2015;24(1):238-44.

19 Silva ACR, Reis SA, Santos PG. Gestão pública hospitalar: um estudo de caso do serviço de urgência e emergência de um hospital de grande porte do Distrito Federal. Rev Gestão Ind. 2017;13(4):70-97.

20 Ruiz FS, Oliveira AF, Simão ANC, Lozovoy MAB, Alfieri DF, Sandrini F, et al. Associação entre deficiência de vitamina $\mathrm{D}$, adiposidade e exposição solar em participantes do sistema de hipertensão arterial e diabetes melito. Semin Ciências Biológicas e da Saúde [Internet]. 2014 [citado em 2018 jul 25];35(2):103-14. Disponível em: http://www.uel.br/ revistas/uel/index.php/seminabio/article/view/19328.

21 Freitas JGA, Nielson SEO, Porto CC. Studies on diabetic patients in primary care. Rev da Soc Bras Clínica Medica. 2015;13(1):75-84. 
22 Jasmim JS, Queluci GC. Studies On Diabetic Patients In Primary Care. J Nurs UFPE on line. 2018;12(4):1072-84.

23 Bündchen DC, Schenkel IC, Santos RZ, Carvalho T. Exercise controls blood pressure and improves quality of life. $2013 ; 19(2): 91-5$.

24 Pinho NA, Pierin AMG. Hypertension Control in Brazilian Publications. Arq Bras Cardiol. 2013;101(3):65-73.

25 Santos MVR, Oliveira DC, Arraes LB, Oliveira DA, Medeiros L, Novaes MA. Adesão ao tratamento anti-hipertensivo: conceitos , aferição e estratégias inovadoras de abordagem. Rev da Soc Bras Clínica Médica [Internet]. 2013 [citado em 2018 jul 25];11(1):55-61. Disponível em: http://www.ncbi. nlm.nih.gov/pubmed/17070434.

26 Teixeira MZ, Lin CA. Educação médica em terapêuticas não convencionais. Rev Med. 2013;92(4):224-35.

27 Pereira MST. O efeito da acupunctura na Hipertensão: estudo prospectivo, randomizado, controlado [dissertação]. Portugal: Instituto de Ciências Biomédicas Abel Salazar, Universidade do Porto; 2015.
28 Li J, Wang Y, He K, Peng C, Wu P, Li C, et al. Effects of acupuncture at LR3 on cerebral glucose metabolism in a rat model of hypertension: A 18F-FDG-PET study. Evid Based Complement Altern Med. 2018;2018:1-8.

29 Zheng H, Han Y, Du Y, Shi X, Huang H, Yu X, et al. Regulation of Hypertension for Secondary Prevention of Stroke: The Possible 'Bridging Function" of Acupuncture.' Complement Med Res. 2018;25(1):45-51.

30 Frezza SC. Ansiedade, estresse e auriculoterapia: uma revisão de literatura [dissertação]. Criciúma (SC): Universidade do Extremo Sul Catarinense - UNESC; 2016.

31 Carmo MA, Antoniassi DP. Avaliação da dor e qualidade de vida em mulheres com fibromialgia submetidas ao tratamento de auriculoterapia associada à fisioterapia ou exercícios físicos. Rev Bras Qual Vida. 2018;10(1):1-17. 
